\title{
Mathematical Modeling of Cardiovascular System Dynamics Using a Lumped Parameter Method
}

\author{
Eun Bo SHIM, Jong Youb SAH ${ }^{*}$, and Chan Hyun Youn ${ }^{\dagger}$ \\ Division of Mechanical \& Biomedical Engineering, Kangwon National University, Hyoja-dong, Chuncheon, \\ Kangwon-do 200-701, Republic of Korea; *Department of Mechanical Engineering, Kyungsan, Kyungsangbuk-do, \\ Yeungnam University, Republic of Korea; and `Department of Engineering, Information and Communications \\ University, Taejeon, Republic of Korea
}

\begin{abstract}
This work reviews the main aspects of cardiovascular system dynamics with an emphasis on modeling hemodynamic characteristics by the use of a lumped parameter approach. The methodological and physiological aspects of the circulation dynamics are summarized with the help of existing mathematical models. The main characteristics of the hemodynamic elements, such as the heart and arterial and venous systems, are first described. Distributed models of an arterial network are introduced, and their characteristics are compared with those of lumped
\end{abstract}

parameter models. We also discuss the nonlinear characteristics of the pressure-volume relationship in veins. Then the control pathways that participate in feedback mechanisms (baroreceptors and cardiopulmonary receptors) are described to explain the interaction between hemodynamics and autonomic nerve control in the circulation. Based on a set-point model, the computational aspects of reflex control are explained. [The Japanese Journal of Physiology 54: 545-553, 2004]

Key words: lumped parameter model, dynamics of the cardiovascular system, autonomic nerve control.

\section{Introduction}

The cardiovascular system is responsible for supplying the tissues and organs of the body with oxygen and other nutrients required for their metabolic needs, and its proper functioning is critical for an individual's optimal performance. Cardiovascular disease is a major cause of death in humans. Many experimental studies have examined the mechanisms and therapies of cardiovascular diseases. Along with the experimental approach, mathematical modeling has become a popular way to analyze the cardiovascular system.

Many mathematical models of the cardiovascular system have been published since Grodins [1] made the first global dynamic model of one in 1959. There is a wide variety of such models, which depends on their purpose and the methodology used [2-10]. Researchers have approached the modeling problem from several different perspectives. Some subjects that have been extensively studied include hemodynamic models of specific vascular beds, such as the coronary or cerebral circulation [11-15]; the distributed impedance of arterial and pulmonary stress $[6,7,16,17]$; lumped models of the integrated cardiovascular system $[5,9,18,19]$; and hemodynamic control system $[8,20]$.

This paper reviews the fundamental issues in the development of lumped models of the integrated circulatory system combined with short-term nerve control. The models, related with the response to thermal stress, are not considered here because of the complexity of the underlying mechanisms. Furthermore, local circulations, such as coronary, cerebral, and renal circulation, are not discussed here. We emphasize the fundamentals and recent progress in models of the overall cardiovascular system, using the lumped

Received on July 17, 2004; accepted on September 22, 2004

Correspondence should be addressed to: Eun Bo Shim, Division of Mechanical \& Bioemdical Engineering, Kangwon National University, Hyoja-dong, Chuncheon, Kangwon-do 200-701, Republic of Korea. Tel: +82-33-250-6318, Fax: +82-33-250-6318, E-mail: ebshim@kangwon.ac.kr 
parameter method combined with an autonomous nerve system for controlling heart mechanics and vessel hemodynamics.

\section{Lumped Parametric Approximation of The Hemodynamic System}

A model-based understanding of the fundamentals of blood flow dynamics, or hemodynamics, is important for the diagnosis and management of diseases of the cardiovascular system, including coronary artery and heart muscle dysfunction, valvular disorders, and pulmonary disease. Models of the cardiovascular system will help in understanding these conditions, ultimately leading to improved treatment. Of several modeling approaches, the lumped parameter model has become a popular way to analyze the hemodynamics of the cardiovascular system.

In general, cardiovascular system models consist of two major parts: the hemodynamic system and the autonomic nerve control, as shown in Fig. 1. The hemodynamics of vessels can be represented by the relationship between blood pressure and blood flow rate in the cardiovascular system. In the lumped model, the hemodynamic elements of the cardiovascular system are expressed as a series of equivalent elements in an electric circuit, as shown in Fig. 2. The application of Kirchhoff's law to each node in the lumped parameter hemodynamic model leads to the following differential equation for mass conservation.

$$
\frac{d V}{d t}=Q_{\text {in }}-Q_{\text {out }}
$$

where

$$
Q=\left(P_{\mathrm{i}}-P_{0}\right) / R
$$

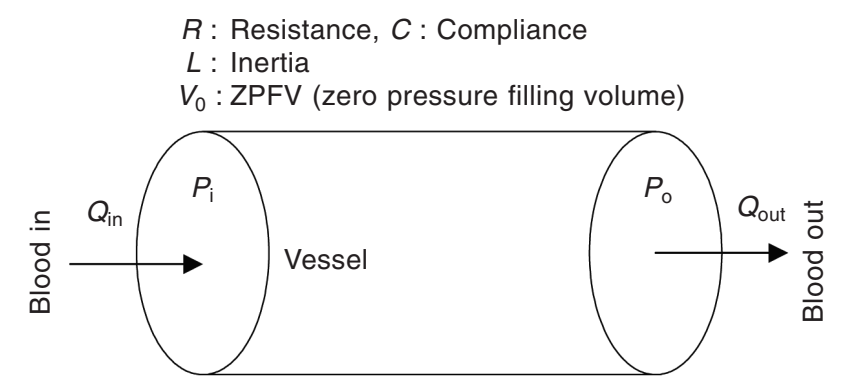

(a) Hemodynamic elements of a blood vessel

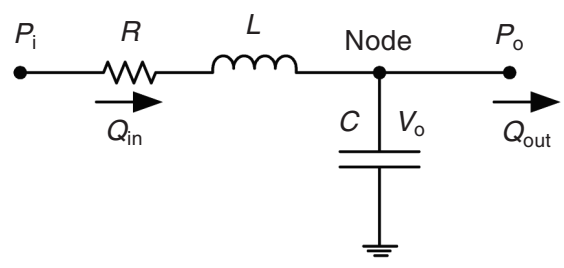

(b) Equivalent electric circuit

Fig. 2. Electrical analog of vascular hemodynamic elements. (a) Hemodynamic elements of a blood vessel. (b) Equivalent electric circuit.

$$
P=V / C
$$

In these equations, $V$ is the volume of a compartment, $Q$ is the flow rate, $P$ is the pressure, and $C$ is the capacitance. A new volume can be calculated from the flow rates derived by using Eq. 2. After obtaining the volume, the pressures at the nodes are calculated by using Eq. 3. The application of this formula to the other nodes enables us to obtain all the lumped information of the cardiovascular hemodynamics. In the remainder of this chapter, we briefly review hemodynamic modeling of circulation elements, using the lumped parameter approach.

Heart modeling. The ventricle in the cardiovascular system can be approximated as a chamber with

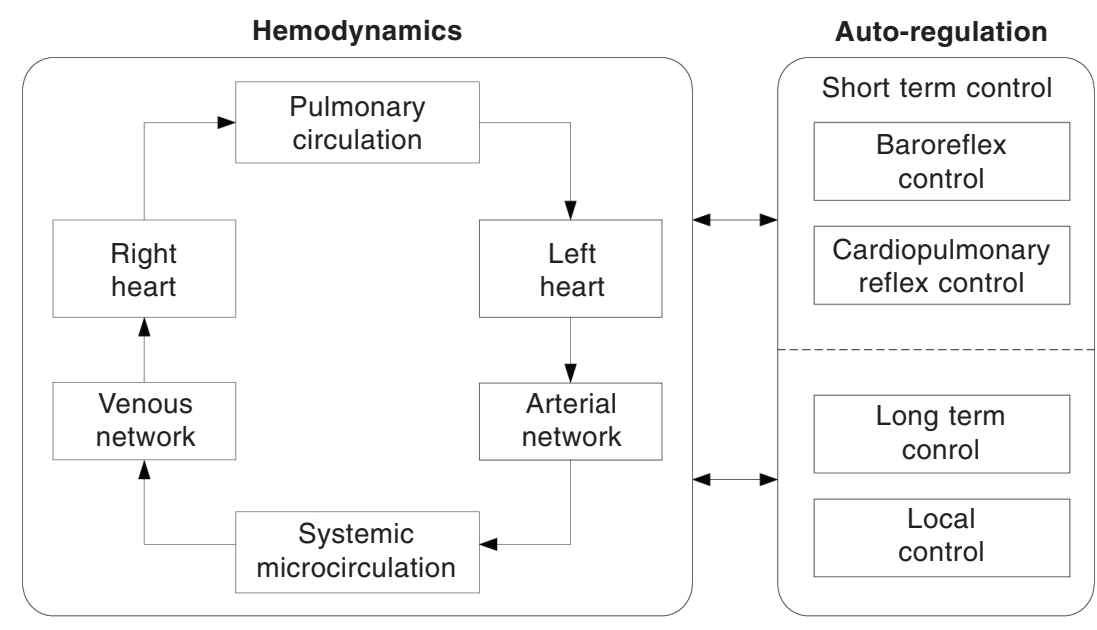

Fig. 1. Schematic of the cardiovascular system. 
an entrance and an exit valve, whose compliance changes as a function of time. The compliance can be replaced by a capacitor in an electric analog. This approach is called the variable capacitor model. In 1982, Campbell et al. [21] developed a five-compartment model of the cardiovascular system with capacitances that vary with time, as proposed by Sunagawa and Sagawa [22]. This method has been used in most of the published cardiovascular system models $[8,22-24]$ because it is relatively simple and easily incorporates experimental results concerning the pressure-volume relation of the ventricle. In this method, the left ventricular capacitance varies between a minimum value during systole and a maximum value during diastole, using predetermined functions that are taken from elastance curves obtained in animal experiments [25].

In terms of the variable capacitor model, both ventricles are characterized by time-dependent compliances that vary between a minimum end-systolic value and a maximum diastolic one according to a predefined function. Estimates of the left ventricular maximum diastolic compliance, $C_{1, \text { diast }}$, are frequently based on estimates of the left ventricular end-diastolic elastance, $E_{1, \text { diast }}\left(E_{1, \text { diast }}=1 / C_{1, \text { diast }}\right)$. The latter is reported to range from 0.15 to $0.6 \mathrm{mmHg} / \mathrm{ml}$ for normal subjects [26]. If directly translated into $C_{1 \text {,diast }}$, these values suggest a maximum diastolic left ventricular capacitance of 1.7 to $6.7 \mathrm{ml} / \mathrm{mmHg}$. The right ventricular maximum diastolic capacitance, $C_{\mathrm{r}, \mathrm{diast}}$, is approximately twice the left ventricular value [27]. The left ventricular end-systolic elastance, $E_{1, \text { sys }}$, is reported to be $2.0 \pm 0.7 \mathrm{mmHg} / \mathrm{ml}$ [28]. When translated into $C_{1, \text { sys }}$, a value close to $0.5 \mathrm{ml} / \mathrm{mmHg}$ seems reasonable [26]. The right ventricular end-systolic capacitance, $C_{\mathrm{r}, \mathrm{sys}}$, ranges from 0.35 to $1.6 \mathrm{ml} / \mathrm{mmHg}$ [27]. The pumping action of the ventricles is realized by varying the ventricular capacitances between $C_{\text {r,diast }}$ and $C_{\mathrm{r}, \text { sys }}$, a $C_{\mathrm{l} \text {,diast }}$ and $C_{\mathrm{l}, \mathrm{sys}}$ [28]. During systole, the right and left ventricular elastances, $E_{\mathrm{r}}(t)$ and $E_{\mathrm{l}}(t)$, change from their respective minimum diastolic value to their end-systolic value according to a halfsine function. During early diastolic relaxation, they change from their respective end-systolic values to their minimum diastolic values according to a halfcosine function. During the remaining diastolic time, the elastances stay at their minimum diastolic values. The ventricular compliances are computed by the use of $C(t)=1 / E(t)$. An example of the left ventricular elastance as a function of time is depicted in Fig. 3 [23] and the data are compared with the experiment [28].

In consideration of the overall cardiovascular system, atrial contraction is not important at normal heart rates, whereas it is critical for adequate ventricular filling at high rates during exercise. Except for studies of the hemodynamic response to exercise, a relatively simple approximation is assumed for atrial function in cardiovascular modeling. In some papers, the atria are not represented explicitly; their function is partially absorbed into the function of the adjacent compartments [23, 24]. Ursino et al. [29-31] assumed noncontracting atria that were approximated simply by using a constant capacitor in their model.

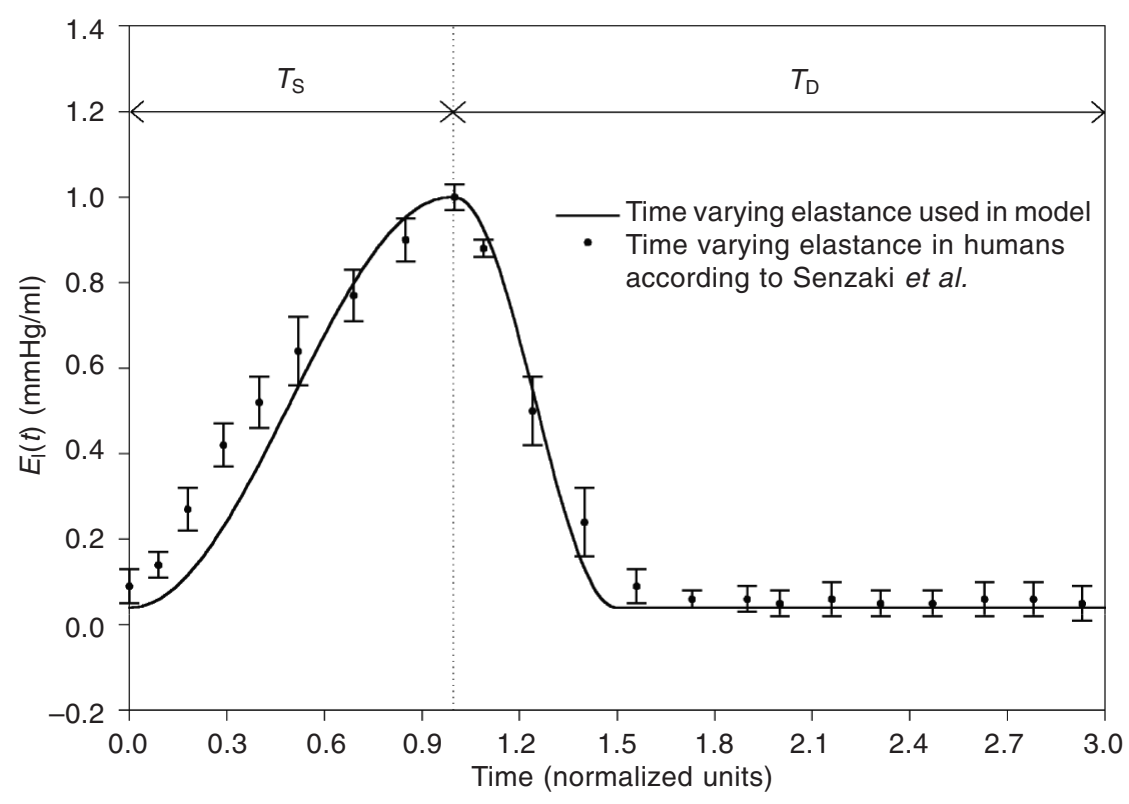

Fig. 3. Left ventricular elastance vs. time. $T_{\mathrm{S}}$ means a systole time duration, whereas $T_{\mathrm{D}}$ represents a diastole time duration. The data generated from the variable capacitor model are compared with the experiment of Serizaki et al. [28]. 
Arterial network. In the lumped parameter model, the average pressure and flow rate are modeled as the electric potential and current, respectively. An arterial vessel is described by using its impedance, which is represented by an appropriate combination of resistors, capacitors, and inductors. The resistors are used to model viscous dissipation, and the capacitors account for artery compliance: the ability to accumulate and release blood by elastic deformation. The inductors are used to model convective inertia terms (Fig. 2).

There are two popular types of models for representing arterial networks in models of the cardiovascular system: lumped parameter models and distributed models. Some models are based on lumped parameter representations of the arterial networks [32-38], and others model vessel hemodynamics by using the fluid dynamic equations that govern flow through a distributed, compliant network [39-42]. Lumped parameter models are generally successful in illuminating the complex interaction of the major hemodynamic elements and the control system. However, their inability to represent the spatial distribution of hemodynamic elements is a severe limitation of their use in clinical studies.

Guyton et al. [36] developed a refined lumped parameter model of the cardiovascular system that embodies the behavior of the interacting cardiovascular, renal, and respiratory systems, including hormones and blood chemistry, using over 350 com- partments. Avolio [3] proposed a lumped parameter model in which a 128-segment arterial network was solved computationally. Stettler et al. [43] developed a distributed representation of the arterial system. Unlike the lumped approximation for the arterial system, they solved the fluid mechanics equations for onedimensional momentum and continuity by using the finite difference method and the method of characteristics (Fig. 4). They also included the effects of wall viscoelasticity and periodic wall shear. Xiao et al. [44] presented a model-based assessment of cardiovascular health by using a noninvasive method of evaluating several of these parameters, including systemic vascular resistance, maximum left ventricular elasticity, end diastolic volume, and cardiac output. The method is based on a distributed arterial model of the human cardiovascular system (28 arterial segments) to generate a solution library. They also used this to simulate the hemodynamics created by enhanced external counterpulsation, which is used to aid a failing heart by sequentially inflating cuffs on the lower extremity, out-of-phase with the left ventricle.

Systemic microcirculation. The term "microcirculation" includes the arterioles, precapillary sphincters, capillaries, and postcapillary venules. It is often assumed that the major resistive component in the systemic circulation is at the level of the microcirculation. In general, the dimension of the resistance is expressed in "peripheral resistance units (PRU: $\mathrm{mmHg} \mathrm{s} / \mathrm{ml})$." The total peripheral resistance of a

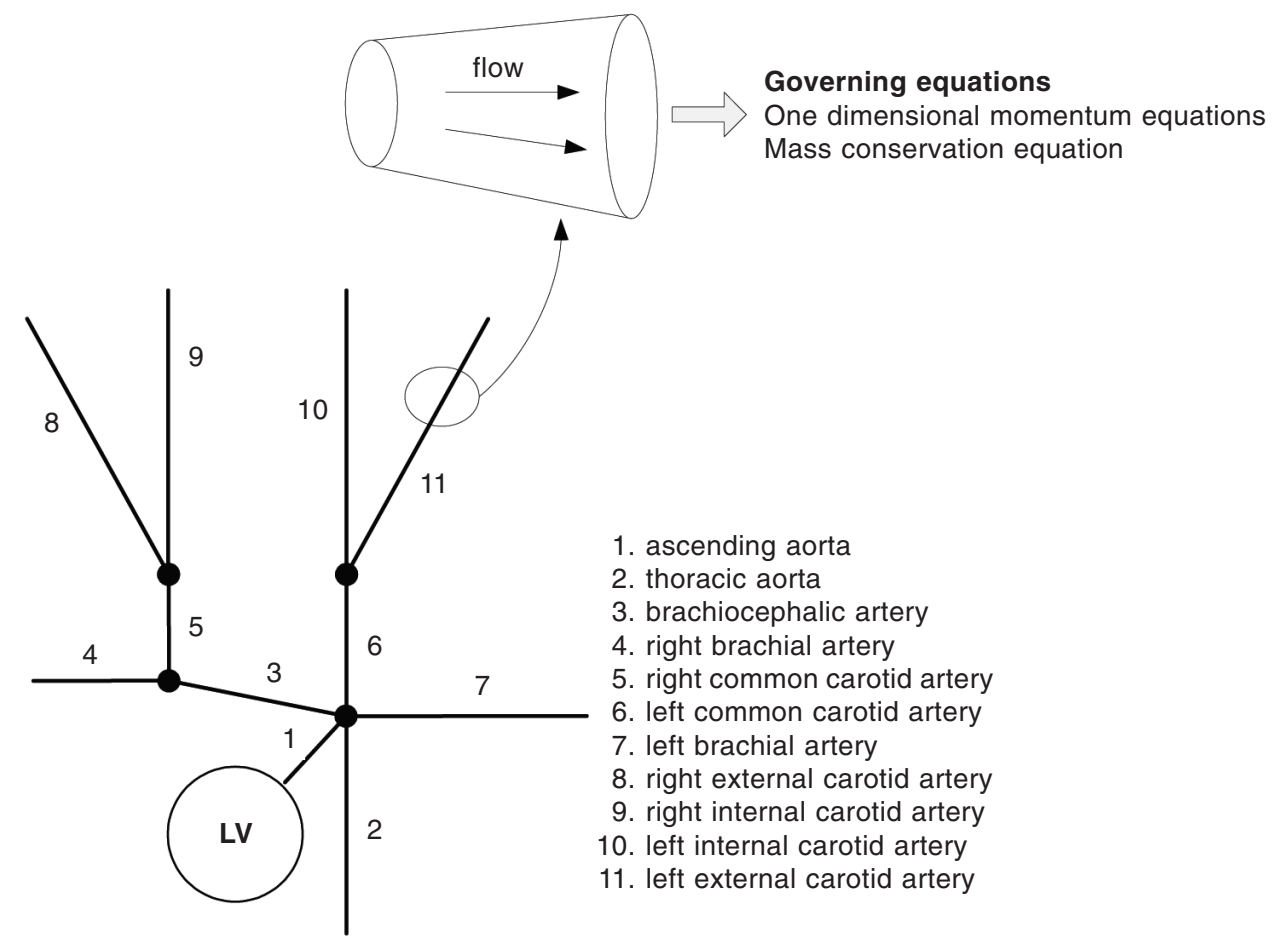

Fig. 4. An example of the distributed representation of the arterial network in the upper body. 
human is close to 1 PRU, which is given as the cardiac output divided by the pressure difference between arteries and veins.

The degree of complexity of microcirculation modeling is dictated by the nature of the questions being asked and the region of the study's focus. For example, some models assume that the microcirculation is a resistor [24, 33] whose value is nearly 1 PRU, and others use a multibranched approximation for the microcirculation $[31,32]$. The microcirculation is especially important in hemodialysis simulation [45-47] and microgravity modeling of the circulation $[23,48]$.

Melchior et al. [25] and Ursino et al. [29] presented a two-branch model in which the microcirculation compartment is divided into two pathways: lower limb and the remaining compartment except this. By contrast, Heldt et al. [23] used a four-branch model of the microcirculation to simulate orthostatic intolerance. They modeled arteriolar resistance to produce a total peripheral resistance of 1.0 PRU based on estimates of the distribution of cardiac output $(\mathrm{CO})$ to the four circulatory branches (upper body [23\% of $\mathrm{CO}$ ], kidney [22\% of $\mathrm{CO}$ ], splanchnic circulation [30\% of $\mathrm{CO}$ ], and lower body [25\% of $\mathrm{CO}]$ ).

In the hemodialysis simulations using a lumped model done by Ursino's group [45-47], mass transfer in the blood-tissue-dialysate system was simulated with a model having three compartments: intracellular, interstitial, and plasma compartments. Fluid exchange between the intracellular and interstitial compartments depends on the osmotic concentration, whereas the hydraulic and osmotic pressure gradients at the capillary compartment determine fluid exchange between the interstitial fluid and plasma. The blood volume change during hemodialysis activates the autonomous nerve control mechanism of the cardiopulmonary reflex resulting from the variation of the returned blood volume to the right atrium.

Venous network and pulmonary circulation. Capillaries unite to form veins, which convey blood back to the heart. To model the venous system with a lumped parameter model, resistance and capacitance values must be approximated. Compared with the systemic microcirculation, the resistance to blood flow in a vein is very low. In a complex model, Beneken [49] used individual peripheral venous resistance values that produced an overall venous resistance of 0.08 PRU. However, the venous transmural pressure in parts of the dependent vasculature can reach levels at which the nonlinear nature of the venous pressure-volume relation becomes important [50]. In particular, the lower limb, splanchnic, and abdominal venous compartments show nonlinear pressure-volume relations. The following equation is one example of a nonlinear relation proposed by Melchior et al. [24]:

$$
\Delta V=\frac{2 \Delta V_{\max }}{\pi} \arctan \left(\frac{\pi C_{0}}{2 \Delta V_{\max }} \Delta P_{\operatorname{tran}}\right)
$$

where $\Delta V$ is the change in compartment volume because of a change in transmural pressure, $\Delta P_{\text {tran }}$. $\Delta V_{\max }$ is the maximal change in compartment volume, and $C_{0}$ is the compartment compliance at baseline transmural pressure. A schematic diagram of this relation is depicted in Fig. 5. An abrupt decrease in venous volume in the curve means vessel collapsing, and this phenomenon has been computed by Luo and Pedley [51] and Shim and Kamm [52].

As far as we know, no distributed model for the venous network has been proposed. However, it is worth noting that a distributed venous model may be more important in studies of venous-related diseases than a distributed arterial model because of the critical role of blood volume shifts between different vascular compartments on the venous side.

The pulmonary vasculature has the comparatively easy task of forcing blood through the low-resistance lung vasculature and returning it to the left heart. In nature, an accurate model of the pulmonary system requires a nonlinear "vascular waterfall" resistance and a nonlinear pulmonary capacitance [53]. Linear models are generally chosen to produce acceptable behavior for the integrated system.

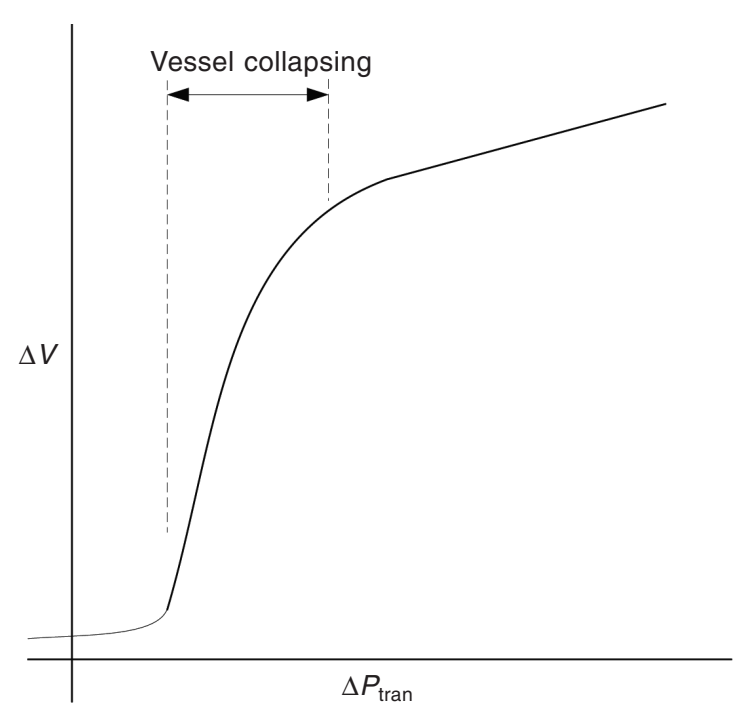

Fig. 5. Pressure-volume relation in veins. 


\section{Short-Term Nerve Control of the Cardiovascular System}

The hemodynamic properties of the cardiovascular system are modulated by a network of homeostasismaintaining feedback mechanisms. This feedback control system must account for the major reflex mechanisms that govern the short-term behavior of the cardiovascular system, i.e., the two major neurally mediated cardiovascular reflexes: the arterial baroreflex and the cardiopulmonary reflex. This review does not consider long-term controls that are concerned with fluid and electrolyte regulation, such as the rennin-angiotensin-aldosterone system, arterial natriuretic peptide, or $\mathrm{ADH}$. Under certain conditions there may be important interactions between chemoreceptors and the baroreceptor reflex, but this is not considered here. A schematic representation of shortterm regulation is depicted in Fig. 6. This figure shows the autonomous nerve control with the baroreceptor reflex and the cardiopulmonary control systems. Locally sensed blood pressures at carotid artery $\left(P_{\mathrm{A}}\right)$ and central vein $\left(P_{\mathrm{V}}\right)$ are relayed to the autonomic nervous system (ANS) where error signals $\left(P_{\mathrm{A}}^{\text {eff }}, P_{\mathrm{A}}^{\text {eff }}\right)$ are generated by subtracting predefined set-point values $\left(P_{\mathrm{A}}^{\text {ref }}, P_{\mathrm{A}}^{\text {ref }}\right)$ from the afferent pressure signals $\left(P_{\mathrm{A}}\right.$, $P_{\mathrm{V}}$ ). These error signals subsequently dictate the efferent activity of the reflex model via parasympathetic and sympathetic arcs, such that the error signals in the following computational steps approach zero.
Baroreceptor reflex control. The baroreflex system consists of the carotid and aortic baroreceptors, which have different sensitivities [54]. However, no published papers include both receptors simultaneously using the lumped parameter approach. These receptors transfer a signal to the autonomic nervous system. A fast parasympathetic reflex via the vagus nerve tightly controls heart rate through the sinoatrial (SA) node, and slower sympathetic fibers modulate the strength of ventricular contraction and peripheral vascular tone besides making a slow contribution to the heart rate.

Many papers have implemented baroreflex control. DeBoer et al. [54] proposed the set-point model of the arterial baroreflex. The short-term control mechanism implemented by Heldt et al. [23] and Shim et $a l$. [24] is based on a set-point model that aims at maintaining a constant mean arterial blood pressure by adjusting the heart rate, peripheral resistance, venous tone, and right and left end-systolic cardiac capacitance dynamically (Fig. 6).

Cardiopulmonary reflex control. Cardiopulmonary receptors, which are in the atria, ventricles, and pulmonary vasculature, provide the controller with information about blood volume. Their effector mechanisms include varying arteriolar and venous tone, but with anatomic patterns that differ from the arterial baroreceptors. Melchior et al. [55] first introduced modeling of the cardiopulmonary reflex to simulate orthostatic intolerance. This model, combined

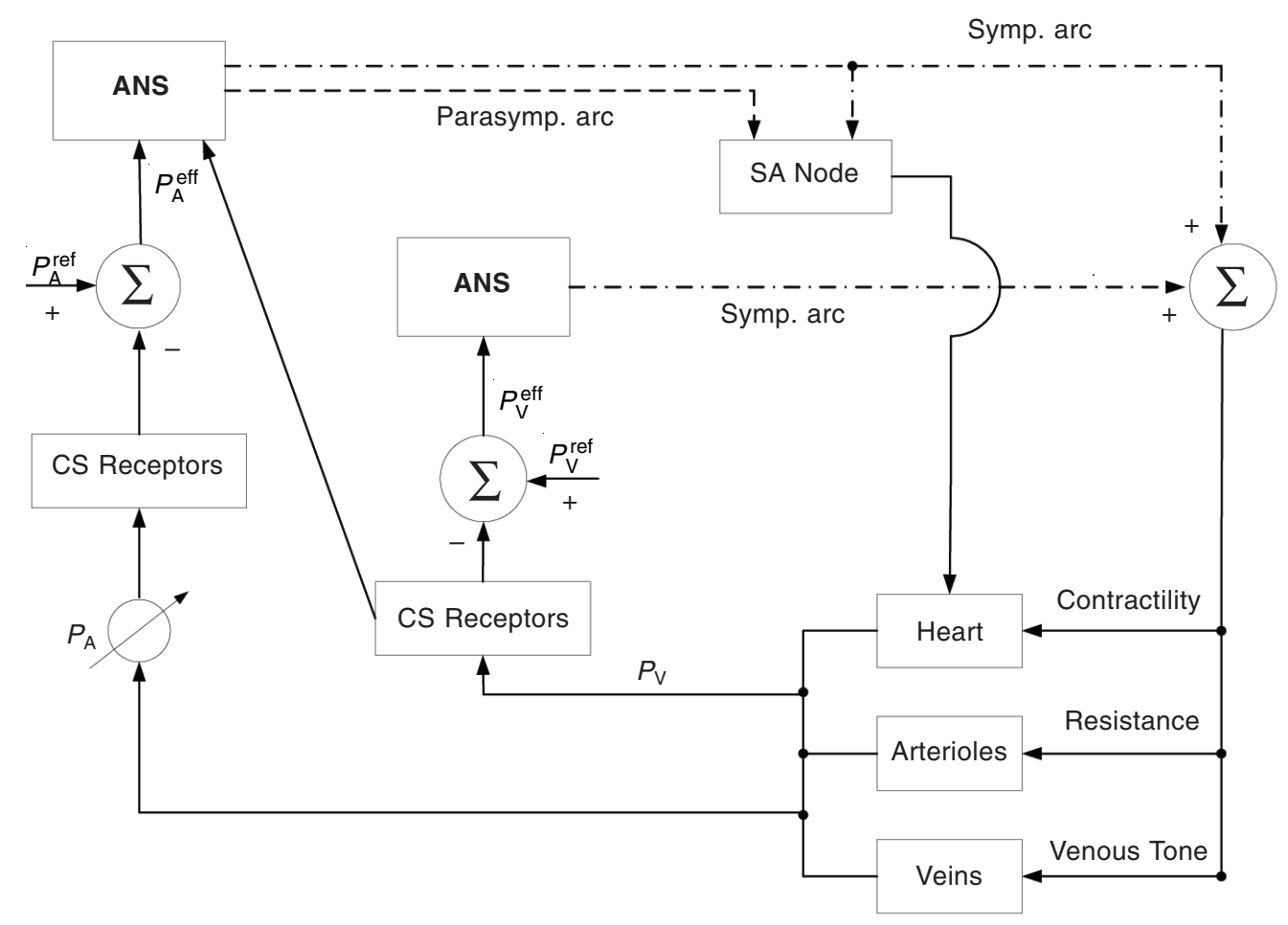

Fig. 6. Control mechanisms, including two pathways. 
with that of the arterial baroreflex, was modified by Ursino et al. [56] to mathematically investigate physiological factors involved in hemodialysis hypotension. Heldt et al. [29] integrated the arterial baroreflex and cardiopulmonary reflex loop in their lumped parameter model. They considered the interaction between them based on the experimental observation that central hypovolemia leads to an increase in the heart rate gain of the arterial baroreflex.

\section{Conclusion}

In this paper we reviewed the main aspects of cardiovascular system dynamics with an emphasis on modeling hemodynamic characteristics by using a lumped parameter approach. The hemodynamic elements, such as the heart and arterial and venous systems, were described. We also discussed the nonlinear characteristics of the pressure-volume relationship in veins. The control pathways that participate in feedback mechanisms (baroreceptors and cardiopulmonary receptors) were described to explain the interaction between hemodynamics and autonomic nerve control in the circulation.

We believe that simulation technology combined with powerful computing resources can make major contributions to assessing the blood hemodynamics in the circulation. Furthermore, experimental results can be interpreted more effectively with the aid of a computational model that simulates the critical components and behaviors of the cardiovascular system. Experiments are used to refine models and specify their parameters. Conversely, models illuminate and enhance the interpretation of the experimental results. We view experiments and computational models as highly synergistic because the value of one is greatly enhanced by the existence of the other. It could be argued that these are not merely advantages, but that they are essential aspects of the study of blood hemodynamics associated with cardiovascular diseases.

This work was supported by Institute of Information Technology Assessment (IITA) through the Grid Middleware Center Project.

\section{REFERENCES}

1. Grodins FS: Integrative cardiovascular physiology: A mathematical synthesis of cardiac and blood vessel hemodynamics. Q Rev Biol 34: 93-116, 1959

2. Attinger EO, and Anne A: Simulation of the cardiovascular system. Ann NY Acad Sci 128: 810-829, 1966

3. Avolio AP: Multi-branched model of the human arterial system. Med Biol Eng Comput 18: 709-718, 1980
4. Dagen J: Pulsatile mechanical and mathematical model of the cardiovascular system. Med Biol Eng Comput 20: 601-607, 1982

5. Hardy HH, Collins RE, and Calvert RE: A digital computer model of the human circulatory system. Med Biol Eng Comput 20: 550-564, 1982

6. LaCourse JR, Mohankrishnan G, and Sivaprasad K: Simulations of arterial pressure pulses using a transmission model. J Biomech 19: 771-780, 1986

7. Sud VK, and Sekhon GS: Analysis of blood flow through a model of the human arterial system under periodic body acceleration. J Biomech 19: 929-941, 1986

8. Ursino M: Interaction between carotid baroregulation and the pulsating heart: a mathematical model. Am J Physiol 275(5 Pt 2): H1733-H1747, 1988

9. Boyers DG., Cuthbertson JG, and Leutscher JA: Simulation of the human cardiovascular system: a model with normal responses to change of posture, blood loss, transfusion, and autonomic blockade. Simulation 18: 197-205, 1972

10. Croston RC, Rummel JA, and Kay FJ: Computer model of cardiovascular control system responses to exercise. J Dynamic Systems Measurement Control 95: 301-307, 1973

11. Ejaz T, Takemae T, Kosugi Y, and Hongo M: The high zero-flow pressure phenomenon in coronary circulation: a simulation study. Front Med Biol Eng 11: 335-340, 2002

12. Kresh JY, Fox M, Brockman SK, and Noordergraaf $A$ Model-based analysis of transmural vessel impedance and myocardial circulation dynamics. Am J Physiol 258 (1 Pt 2): H262-H276, 1990

13. Schreiner W, Neumann F, and Mohl W: Simulation of coronary circulation with special regard to the venous bed and coronary sinus occlusion. J Biomed Eng 12: 429-443, 1990

14. Magosso E, and Ursino M: Modelling study of the acute cardiovascular response to hypocapnic hypoxia in healthy and anaemic subjects. Med Biol Eng Comput 42: 158-166, 2004

15. Ursino M, Magosso E, and Avanzolini G: An integrated model of the human ventilatory control system: the response to hypoxia. Clin Physiol 21: 465-477, 2001

16. Jager $G N$, Westerhof $N$, and Noordergraaf $A$ : Oscillatory flow impedance in electrical analog of arterial system: representation of sleeve effect and nonNewtonian properties of blood. Circ Res 16: 121-133, 1965

17. Porenta G, Young DF, and Rogge DR: A finite element model of blood flow in arteries including taper, branches, and obstructions. J Biomech 108: 161-167, 1986

18. Li X, Bai J, Cui S, and Wang S: Simulation study of the cardiovascular functional status in hypertensive situation. Comput Biol Med 32: 345-362, 2002

19. Masuzawa T, Fukui Y, and Smith NT: Cardiovascular simulation using a multiple modeling method on a digital computer-simulation of interaction between the cardiovascular system and angiotensin II. J Clin Monit 8: 50-58, 1992

20. Jaron D, Moore DW, and Chu CL: Cardiovascular model for studying impairment of cerebral function 
during $+\mathrm{Gz}$ stress. Aviat Space Environ Med 55: 24-31, 1984

21. Campbell K, Zeglen M, Kagehiro T, and Rigas $H$ : A pulsatile cardiovascular computer model for teaching heart-blood vessel interaction. Physiologist 25: 155-162, 1982

22. Sunagawa K, and Sagawa K: Models of ventricular contraction based on time-varying elastance. Crit Rev Biomed Eng 7: 193-228, 1982

23. Heldt T, Shim EB, Kamm RD, and Mark RG: Computational modeling of cardiovascular response to orthostatic stress. J Appl Physiol 92: 1239-1254, 2002

24. Shim EB, Heldt T, Kamm RD, Mark RG, and Youn CH: Computational Modeling of the Cardiovascular System After Fontan Procedure. Lecture Notes in Computer Science 2526: 105-114, 2002

25. Melchior FM, Srinivasan RS, and Charles JB: Mathematical modeling of human cardiovascular system for simulation of orthostatic response. Am J Physiol 262(6 Pt 2): H1920-H1933, 1992

26. Mirsky I: Elastic Properties of the Myocardium: A quantitative approach with physiological and clinical applications. In: Handbook of Physiology (The Cardiovascular System), ed. Berne RM, American Physiological Society, Bethesda, pp 497-431, 1979

27. Dell'Italia LJ, and Walsh RA: Application of a time varying elastance model of right ventricular performance in man. Cardiovasc Res 22: 864-874, 1988

28. Senzaki H, Chen $\mathrm{CH}$, and Kass D: Single-beat estimation of end-systolic pressure-volume relation in humans: a new method with the potential for non-invasive application. Circulation 94: 2497-2506, 1996

29. Ursino M: Interaction between carotid baroregulation and the pulsating heart: a mathematical model. Am J Physiol 275(5 Pt 2): H1733-H1747, 1998

30. Ursino M, and Magosso E: Role of short-term cardiovascular regulation in heart period variability: a modeling study. Am J Physiol Heart Circ Physiol 284: H1479-H1493, 2003

31. Ursino M: A mathematical model of the carotid baroregulation in pulsating conditions. IEEE Trans Biomed Eng 46: 382-392, 1999

32. Burkhoff, DJ, Alexander, and Schipke K: Assessment of Windkessel as a model of aortic impedance. Am J Phys 255 (Heart Circ Physiol 24): H742-H753, 1988

33. Campbell K, Zeglen M, Kagehiro T, and Rigas $H$ : A pulsatile cardiovascular model for teaching heartblood vessel interaction. Physiologist 25: 155-162, 1982

34. Davis TL, and Mark RG: Teaching Physiology Through Simulation of Hemodynamics. Computers in Cardiology 1990, IEEE Computer Society

35. Davis TL: Teaching Physiology Through Interactive Simulation of Hemodynamics, in Department of Electrical Engineering and Computer Science, Massachusetts Institute of Technology, Cambridge, 1991

36. Guyton AC, Coleman TG, Manning, Jr. Rd, and Hall JE: Some problems and solutions for modeling overall cardiovascular regulation. Math Biosci 72: $141-155,1984$
37. Kenner T: Physical and mathematical modeling in cardiovascular systems. In: Quantitative Cardiovascular Studies, University Park, Baltimore, MD, pp 41-109, 1979

38. White RJ, Fitzjerrell DG, and Croston RD: Fundamentals of Lumped Compartment Modelling of the Cardiovascular System, in Adv. Cardiovasc. Phys., Karger, Basel, pp 162-184, 1983

39. Braakman R, Sipkema P, and Westerhof N: A dynamic nonlinear lumped parameter model for skeletal muscle circulation. Ann Biomed Eng 17: 593-616, 1989

40. Rideout VC, and J Katra: Computer simulation study of the pulmonary circulation. Simulation 12: 239-245, 1969

41. Wang JZ, Tie B, Welkowitz W, Kostis J, and Semmlow $\mathrm{J}$ : Incremental network analogue model of the coronary artery. Med Biol Eng Comput 27: 416-422, 1989

42. Zagzoule M, and Marc-Vergnes JP: A global mathematical model of the cerebral circulation in man. $J$ Biomech 19: 1015-1022, 1986

43. Stettler JC, Niederer P, and Anliker M: Theoretical analysis of arterial hemodynamics including the influence of bifurcations. Part 1: mathematical models and prediction of normal pulse patterns. Ann Biomed Eng 9: 145-164, 1981

44. Xiao X, Ozawa ET, Huang Y, and Kamm RD: Modelbased assessment of cardiovascular health from noninvasive measurements. Ann Biomed Eng 30: 612-623, 2002

45. Ursino M, Coli L, Brighenti C, Chiari L, de Pascalis A, and Avanzolini G: Prediction of solute kinetics, acidbase status, and blood volume changes during profiled hemodialysis. Ann Biomed Eng 28: 204-216, 2000

46. Coli L, Ursino M, De Pascalis A, Brighenti C, Dalmastri V, La Manna G, Isola E, Cianciolo G, Patrono D, Boni $\mathrm{P}$, and Stefoni S: Evaluation of intradialytic solute and fluid kinetics. Setting up a predictive mathematical model. Blood Purif 18: 37-49, 2000

47. Coli L, Bonomini M, La Manna G, Dalmastri V, and Ursino $\mathrm{M}$, Ivanovich $\mathrm{P}$, and Bonomini V. Clinical use of profiled hemodialysis. Artif Organs 22: 724-730, 1998

48. Melchior FM, Srinivasan RS, Ossard G, and Clere J$\mathrm{M}$ : A mathematical model of the cardiovascular response to $+G z$ acceleration. Physiologist 36 (1 Suppl): S62-S63, 1993

49. Beneken JEW, and DeWitt B: A physical approach to hemodynamic aspects of the human cardiovascular system. In Physical Bases of Circulatory Transport: Regulation and Exchange, Saunders, Philadelphia, pp 1-45, 1967

50. Snyder MF, and Rideout VC: Computer simulation studies of the venous circulation. IEEE Trans Bio-Med Eng 16: 325-334, 1969

51. Luo $X Y$, and Pedley TJ: A numerical simulation of unsteady flow in a 2-D collapsible channel. J Fluid Mech 314: 191-225, 1996

52. Shim EB, and Kamm RD: Numerical simulation of steady flow in a compliant tube or channel with tapered wall thickness, J Fluids Struct 16: 1009-1027, 2002 
53. Thomas JD, Zhou J, Greenberg N, Bibawy G, McCarthy PM, and Vandervoort PM: Physical and physiological determinants of pulmonary venous flow: numerical analysis. Am J Physiol 272(5 Pt 2): $\mathrm{H} 2453-\mathrm{H} 2465,1997$

54. DeBoer RW, Karemaker JM, and Strackee J: Hemodynamic fluctuations and baroreflex sensitivity in humans: a beat- to-beat model. Am J Physiol 253(3 Pt 2): H680-H689, 1987
55. Melchior FM, Srinivasan RS, and Clere JM: Mathematical modeling of human cardiovascular response to LBNP. Physiologist 35 (1 Suppl): S204-S205, 1994

56. Ursino M, Coli L, Brighenti C, Chiari L, de Pascalis $A$, and Avanzolini G: Prediction of solute kinetics, acidbase status, and blood volume changes during profiled hemodialysis. Ann Biomed Eng 28: 204-216, 2000 